\title{
Ubrogepant: First Approval
}

\author{
Lesley J. Scott ${ }^{1}$
}

Published online: 5 February 2020

(c) Springer Nature 2020, corrected publication 2020

\begin{abstract}
Ubrogepant (Ubrelvy ${ }^{\mathrm{TM}}$ ) is an orally administered, small molecule, highly-selective, calcitonin gene-related peptide (CGRP) antagonist that was developed by Allergan under license to Merck \& Co. as an acute treatment for migraine. In December 2019, ubrogepant received its first global approval in the USA for the acute treatment of migraine ( \pm aura) in adults. This article summarizes the milestones in the development of ubrogepant leading to its first global approval for the acute treatment of migraine ( \pm aura) in adults.
\end{abstract}

\section{Ubrogepant (Ubrelvy ${ }^{\mathrm{TM}}$ ): Key points}

A calcitonin gene-related peptide receptor antagonist was being developed by Allergan under license to Merck $\&$ Co. for the acute treatment of migraine

Received its first approval on 23 December 2019 in the USA

Approved for use in the acute treatment of migraine ( \pm aura) in adults

\section{Introduction}

Calcitonin gene-related peptide (CGRP), a vasodilatory neuropeptide involved in nociceptive transmission and modulation, and its receptors are widely expressed in central and peripheral regions of the nervous system [1-3]. Extensive

Enhanced material for this AdisInsight Report can be found at https://doi.org/10.6084/m9figshare.11626044.

This profile has been extracted and modified from the AdisInsight database. AdisInsight tracks drug development worldwide through the entire development process, from discovery, through preclinical and clinical studies to market launch and beyond.

Lesley J. Scott

dru@adis.com

1 Springer Nature, Private Bag 65901, Mairangi Bay, Auckland 0754, New Zealand evidence supports the important role that CGRP plays in migraine pathophysiology, making CGRP and its receptors a novel therapeutic target for the treatment of migraine. Indeed, the recent development of agents that target CGRP and its receptors represent an important advance in the management paradigm for migraine [1-3].

Ubrogepant (Ubrelvy ${ }^{\mathrm{TM}}$ ), a highly potent, orally administered small molecule, is a CGRP receptor antagonist being developed by Allergan under license from Merck \& Co., for the acute treatment of migraine [4]. On the 23 December 2019, the US FDA approved ubrogepant for the acute treatment of migraines ( \pm aura) in adults [5]. It is the first drug in the class of oral CRGP antagonists approved for the acute treatment of migraine [5]. The recommended dosage of oral ubrogepant is $50 \mathrm{mg}$ or $100 \mathrm{mg}$ taken as needed without regard to food [6]. A second dose may be administered at least $2 \mathrm{~h}$ after the initial dose if needed, with a maximum dose in a $24-\mathrm{h}$ period of $200 \mathrm{mg}$. In patients with severe hepatic or renal impairment, the recommended dose is $50 \mathrm{mg}$; a second dose may be administered at least $2 \mathrm{~h}$ after the initial dose if needed [6].

\subsection{Company Agreements}

In July 2015, Merck \& Co. entered into a licensing agreement with Allergan to divest the worldwide rights of small molecule CGRP receptor antagonists, atogepant and ubrogepant. According to the terms of the signed agreement, Allergan will disburse Merck \& Co. an upfront payment of \$US250 million. Merck \& Co. is entitled to receive development and commercial milestone payments, as well as tiered double-digit royalties based on commercialization of the 


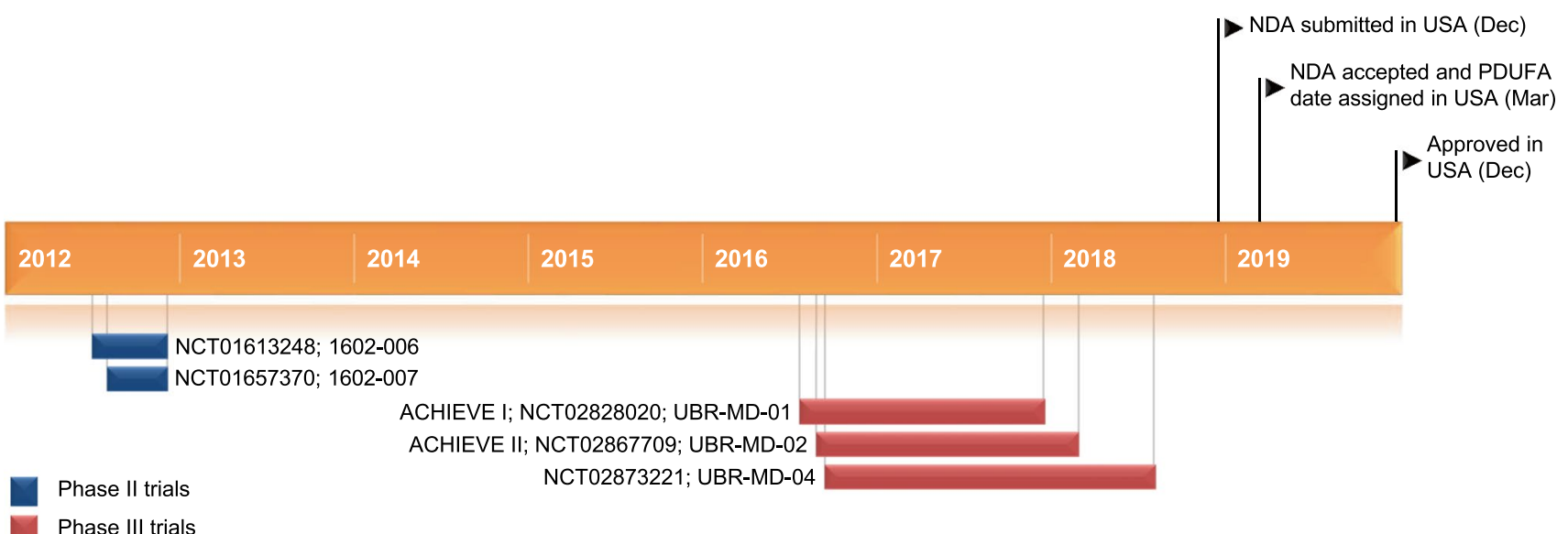

Key milestones in the development of ubrogepant for the acute treatment of migraine. NDA New Drug Application, PDUFA Prescription Drug User Fee Act

programs. Allergan is completely responsible for development, manufacturing and commercialization upon approval and launch of the products [4].

\section{Scientific Summary}

\subsection{Pharmacodynamics}

Ubrogepant is a potent, highly-selective, competitive CGRP receptor antagonist. In functional assays, ubrogepant exhibited similar high-affinity binding for native CGRP receptors [inhibitory constant $\left(\mathrm{K}_{\mathrm{i}}\right) 0.067 \mathrm{nmol} / \mathrm{L}$ ] and for cloned human and rhesus monkey CGRP receptors $\left(\mathrm{K}_{\mathrm{i}} 0.070\right.$ and $0.079 \mathrm{nmol} / \mathrm{L}$ at respective cloned receptors). Ubrogepant demonstrated potent inhibition of the human $\alpha$-CGRPstimulated cyclic AMP response in human CGRP receptorexpressing HEK293 cells (50\% inhibitory concentration of $0.08 \mathrm{nmol} / \mathrm{L}$ ). Relative to other receptors in the calcitonin receptor family, ubrogepant exhibited highly selective antagonist activity against CGRP receptors [7].

Therapeutic concentrations of ubrogepant did not induce major vasoconstrictor effects in cultured human coronary, cerebral and middle meningeal artery in vitro. Ubrogepant exhibited competitive inhibition of $\alpha$-CGRP-induced relaxations, with antagonism of CGRP-induced relaxation more potent for cranial (middle meningeal and cerebral) than coronary arteries [8].

At doses twice the maximum recommended daily dose, ubrogepant does not prolong the QT interval to any clinically relevant extent [6], based on results of thorough QT study in healthy adults [9].

\subsection{Pharmacokinetics}

Ubrogepant exhibits dose-proportional pharmacokinetics and is rapidly absorbed after oral administration, with peak plasma concentrations attained at $\approx 1.5 \mathrm{~h}$. There are no clinically relevant effects of food on the pharmacokinetics of ubrogepant. The drug is $87 \%$ bound to plasma protein in vitro. After a single oral dose, the mean apparent central volume of distribution of ubrogepant is $\approx 350 \mathrm{~L}$ [6].

The primary route of metabolism is via CYP3A4, with the parent compound and two glucuronide conjugate metabolites the most prevalent circulating components. The glucuronide metabolites were $\approx 6000$-fold less potent in the CGRP receptor binding assay and thus, are not expected to contribute to the pharmacological activity of ubrogepant. The elimination half-life of ubrogepant is $\approx 5$ to $7 \mathrm{~h}$. Ubrogepant is primarily eliminated via the biliary/faecal route, with renal<smiles>C[C@H]1[C@H](c2ccccc2)C[C@@H](NC(=O)c2cnc3c(c2)C[C@@]2(C3)C(=O)Nc3ncccc32)C(=O)N1CC(F)(F)F</smiles>

Chemical structure of ubrogepant 
elimination a minor route $(42 \%$ and $6 \%$ of a radiolabeled dose recovered as parent compound in the faeces and urine, respectively) [6].

There were no clinically relevant effects of age, sex, race, bodyweight, mild or moderate renal impairment, and mild or moderate hepatic impairment on the pharmacokinetics of ubrogepant, based on a population pharmacokinetic (PPK) analyses. Patients with severe renal impairment (estimated glomerular filtration rate $<30 \mathrm{~mL} / \mathrm{min}$ ) have not been studied; dose adjustment is recommended based on absorption, distribution, metabolism and elimination (ADME) information and a conservative assumption that severe renal impairment is unlikely to cause a more than twofold increase in exposure to ubrogepant. No dosing recommendations can be made for patients with end-stage renal disease (creatinine clearance $<15 \mathrm{~mL} / \mathrm{min}$ ). In patients with severe hepatic impairment (Child-Pugh Class C), exposure to ubrogepant increased by $115 \%$; dosage adjustments are required in this population [6].

In vitro, ubrogepant does not inhibit CYP1A2, CYP2B6 or CYP3A4 and is a weak inhibitor of CYP2C8, CYP2C9, CYP2D6, CYP2C19, MAO-A and UGT1A1; this inhibition is not expected to be clinically relevant. At clinically relevant concentrations, ubrogepant is not an inducer of CYP1A2, CYP2B6 or CYP3A4. The drug is a substrate of BCRP and P-glycoprotein (P-gp) transporters in vitro; thus, use of inhibitors of BCRP and/or P-gp may increase exposure to ubrogepant, with dose adjustments of ubrogepant recommended when coadministered with BCRP and/or P-gp only inhibitors (e.g. quinidine, carvedilol, eltrombopag, curcumin). Ubrogepant is a weak substrate of OATP1B1, OATP1B3 and OAT1 transporters, but is not a substrate for OAT3 in vitro. It does not inhibit P-gp, BCRP, BSEP, MRP3, MRP4, OAT1, OAT3 or NTCP transporters, but is a weak inhibitor of OATP1B1, OATP1B3 and OCT2 transporters. No clinically relevant drug interactions are expected with these transporters, except for BCRP and P-gp inhibitors [6].

In healthy volunteers, no clinically relevant pharmacokinetic interactions were observed when ubrogepant was co-administered with oral contraceptives (containing norgestimate and ethinyl estradiol) [10], acetaminophen [11], naproxen [11], sumatriptan [12] or esomeprazole [6]. Coadministration of ubrogepant with ketoconazole (strong CYP3A4 inhibitor) resulted in a significant increase in exposure to ubrogepant; hence, concomitant use of ubrogepant with strong CYP3A4 inhibitors (e.g. ketoconazole, itraconazole, clarithromycin) is contraindicated [6]. Concomitant use of ubrogepant with verapamil (a moderate CYP3A4 inhibitor) increased exposure to ubrogepant; adjustment of the ubrogepant dose is recommended with concomitant use of ubrogepant and moderate CYP3A4 inhibitors (e.g. cyclosporine, ciprofloxacin, fluconazole, fluvoxamine, grapefruit juice). Exposure to ubrogepant was significantly reduced when the drug was coadministered with rifampin (a strong CYP3A4 inducer); thus, concomitant use of ubrogepant with strong CYP3A4 inducers (e.g. rifampin, phenytoin, barbiturates, St. John's wort) should be avoided. Dose adjustment of ubrogepant is recommended when the drug is coadministered with moderate or weak CYP3A4 inducers [6]. Features and properties of ubrogepant

\begin{tabular}{|c|c|}
\hline Alternative names & Ubrogepant; Ubrelvy ${ }^{\mathrm{TM}}$; MK-1602 \\
\hline Class & Amides, antimigraines, fluorine compounds, small molecules, spiro compounds \\
\hline Mechanism of Action & Calcitonin gene-related peptide receptor antagonists \\
\hline Route of Administration & Oral \\
\hline Pharmacodynamics & Potent, highly-selective, competitive calcitonin gene-related peptide receptor antagonist \\
\hline Pharmacokinetics & $\begin{array}{l}\text { Rapidly absorbed; primarily metabolized by CYP3A4; elimination half-life } \approx 5 \text { to } 7 \mathrm{~h} \text {; mainly eliminated } \\
\text { via the biliary faecal route, with minor elimination via the renal route }\end{array}$ \\
\hline \multicolumn{2}{|l|}{ Adverse events } \\
\hline Most frequent $(\geq 2 \%$ and $>$ placebo $)$ & Nausea and somnolence; generally well tolerated \\
\hline \multicolumn{2}{|l|}{ ATC codes } \\
\hline WHO ATC code & N02C (antimigraine preparations) \\
\hline EphMRA ATC code & $\mathrm{N} 2 \mathrm{C}$ (anti-migraine preparations) \\
\hline Chemical Name & $\begin{array}{l}\text { (3S)-N-[(3S,5S,6R)-6-methyl-2-oxo-5-phenyl-1-(2,2,2-trifluoroethyl)piperidin-3-yl]-2-oxospiro[1H- } \\
\text { pyrrolo[2,3-b]pyridine-3,6'-5,7-dihydrocyclopenta[b]pyridine]-3'-carboxamide }\end{array}$ \\
\hline
\end{tabular}




\subsection{Therapeutic Trials}

In pivotal randomized, double-blind, multicentre, phase III trials (ACHIEVE I [13] and II [14]) in adults with moderate to severe migraine, recommended doses of ubrogepant (50 or $100 \mathrm{mg}$ ) were significantly more effective than placebo for the proportion of patients achieving the co-primary endpoints of freedom from pain $2 \mathrm{~h}$ after the initial dose and the absence of the most bothersome symptom (MBS; patientspecified MBS out of photophobia, phonophobia or nausea) associated with migraine at $2 \mathrm{~h}$ postdose. In ACHIEVE I, significantly (all $p \leq 0.002$ ) more patients in the ubrogepant $50 \mathrm{mg}$ and $100 \mathrm{mg}$ groups experienced freedom from migraine pain at $2 \mathrm{~h}$ postdose than placebo recipients (19.2\% and $21.2 \%$ vs $11.8 \%$, respectively; $n=422,448$ and 456 ), with absence of the most bothersome migraine-associated symptom at $2 \mathrm{~h}$ postdose achieved by $38.6 \%, 37.7 \%$ and $27.8 \%$ of patients (both $p=0.002$ vs placebo; $n=420,448$ and 454) [13]. In ACHIEVE II, significantly more patients in the ubrogepant $50 \mathrm{mg}$ than placebo group experienced freedom from pain at $2 \mathrm{~h}$ postdose ( $21.8 \mathrm{vs} 14.3 \% ; p=0.01$; $n=464$ and 456) and absence of the MBS associated with migraine at $2 \mathrm{~h}$ postdose ( 38.9 vs $27.4 \% ; p=0.01 ; n=463$ and 456) [14].

Secondary endpoints for pain relief also generally favoured ubrogepant 50 or $100 \mathrm{mg}$ over placebo treatment, including the percentage of patients with pain relief at $2 \mathrm{~h}$ $(\approx 61.6 \mathrm{vs} \approx 48.7 \%$ of patients in individual treatment groups across trials; all $p \leq 0.01$ vs placebo), sustained pain relief from 2 to $24 \mathrm{~h}(\approx 37.0 \mathrm{vs} \approx 20.9 \%$; all $p \leq 0.01$ vs placebo) and sustained pain freedom from 2 to $24 \mathrm{~h}(\approx 14.2 \mathrm{vs} \approx 8.4 \%$; significance not evaluated for ubrogepant $50 \mathrm{mg}$ vs placebo in ACHIEVE I in accordance with the hierarchical statistical testing plan; $p \leq 0.01$ vs placebo for ubrogepant $100 \mathrm{mg}$ in ACHIEVE I and ubrogepant $50 \mathrm{mg}$ in ACHIEVE II) $[13,14]$. In both studies, the incidence of photophobia and phonophobia was reduced after treatment with ubrogepant $50 \mathrm{mg}$ or $100 \mathrm{mg}$ compared with placebo $[6,13,14]$.

Based on pooled analyses of the $50 \mathrm{mg}$ dose across ACHIEVE I and II, the onset of pain relief was significant at $1 \mathrm{~h}$ in ubrogepant versus placebo recipients [odds ratio (OR) 1.30; $p=0.01]$ [15] and ubrogepant was effective in patients for whom triptans were ineffective [16] (abstracts). In patients who took an optional per-protocol specified second dose of ubrogepant $50 \mathrm{mg}$ or placebo (re-randomization of ubrogepant $50 \mathrm{mg}$ recipients) after an inadequate response to the initial dose of ubrogepant $50 \mathrm{mg}$, significantly more ubrogepant than placebo recipients achieved freedom from pain $2 \mathrm{~h}$ after the second dose (34 vs 19\%; OR $2.85 ; p<0.0001 ; n=156$ and 131 ), based on pooled data (abstract) [17]. In patients achieving freedom from pain $2 \mathrm{~h}$ after the initial dose and who took an optional second dose, this response rate was also significantly better in the ubrogepant than placebo group (55 vs 33\%; OR 2.85; $p<0.0001$; $n=75$ and 57) [17].

In ACHIEVE I and II, participants were aged 18-75 years, had at least a 1-year history of migraine ( \pm aura) that met specified International Classification of Headache Criteria, had migraine onset before 50 years of age and a history of 2-8 migraines with moderate to severe headache pain in each of the 3 months prior to screening. Patients also had to have a history of migraine typically lasting $48-72 \mathrm{~h}$ if treated successfully or unsuccessfully and migraine episodes separated by $\geq 48 \mathrm{~h}$ of headache pain freedom $[13,14]$. Patients were randomized to ubrogepant $50 \mathrm{mg}$ or $100 \mathrm{mg}$ or placebo in ACHIEVE I [13] and to ubrogepant $50 \mathrm{mg}$ or placebo in ACHIEVE II (also had an ubrogepant $25 \mathrm{mg}$ arm which is not discussed) [14]. In both trials, participants were instructed to treat a migraine with moderate to severe headache pain intensity. A second dose of study medication (ubrogepant or placebo) or the patient's usual acute treatment for migraine (included acetaminophen, NSAIDs, opioids, anti-emetics or triptans [14]) was allowed between 2 to $48 \mathrm{~h}$ after the initial treatment for non-responding or recurrent migraine headache [13, 14]. Among patients who selected a MBS, photophobia was selected as the MBS by $56 \%$ of patients, phonophobia by $24 \%$ and nausea by $19 \%$ [6].

Evidence from ACHIEVE I and II are supported by data from a randomized, dose-finding, double-blind, multicentre phase IIb trial (NCT01613248) in adults with migraine [18]. An integrated exposure-response modeling analysis of this phase IIb study and another phase IIb study (NCT01657370) predicted that a dose of ubrogepant $25 \mathrm{mg}$ or higher was likely to achieve significantly better efficacy than placebo with desirable efficacy levels. These data supported the ubrogepant dose selection utilized in the pivotal phase III trials [19].

The beneficial effects of ubrogepant $(50$ or $100 \mathrm{mg}$ doses) observed in the short-term ACHIEVE trials were maintained during the 1-year open-label extension study (NCT02873221; $n=808$ modified intent to treat participants) [abstract] [20]. Over the 1-year period, pain freedom at $2 \mathrm{~h}$ after the initial dose occurred in $\approx 24 \%$ of ubrogepanttreated $(50$ or $100 \mathrm{mg}$ ) migraine attacks and pain relief at $2 \mathrm{~h}$ postdose occurred in $\approx 67 \%$ of ubrogepant-treated attacks. During this 1-year period, 21,454 migraine attacks were treated with 31,968 doses of ubrogepant and an optional second dose of ubrogepant was taken for $\approx 35 \%$ of attacks. Participants were randomized equally to open-label usual care or ubrogepant $50 \mathrm{mg}$ or $100 \mathrm{mg}$, as required for migraine attacks [20]. 
Key clinical trials of ubrogepant for the acute treatment of migraine in adults (Allergan)

\begin{tabular}{lllll} 
Drug(s) & Phase & Status & Location(s) & Identifier \\
\hline Ubrogepant vs placebo & II & Completed & USA & NCT01657370; 1602-007 \\
\hline Ubrogepant vs placebo & II & Completed & USA & NCT01613248; 1602-006 \\
\hline Ubrogepant vs placebo & III & Completed & USA & NCT02828020; ACHIEVE I; UBR-MD-01 \\
Ubrogepant vs placebo & III & Completed & USA & NCT02867709; ACHIEVE II; UBR-MD-02 \\
Ubrogepant vs placebo & III & Completed & USA & NCT02873221; UBR-MD-04
\end{tabular}

\subsection{Adverse Events}

Ubrogepant was generally well tolerated in adults with migraine participating in phase I [21, 22], II [18] and III $[13,14]$ trials. Based on pooled data from ACHIEVE I and II, the most commonly (frequency $\geq 2 \%$ and at a frequency greater than placebo) reported adverse reactions occurring in the ubrogepant $50 \mathrm{mg}(n=954)$, ubrogepant $100 \mathrm{mg}$ $(n=485)$ and placebo $(n=984)$ groups were nausea $(2,4$ and $2 \%$ of patients, respectively), somnolence (2, 3 an $1 \%$; includes sedation and fatigue) and dry mouth $(<1,2$ and $1 \%)$ [6]. Within the 48-h period postdose, 107 of 954 (11.5\%) ubrogepant-treated (50 $\mathrm{mg}$ dose) patients and 113 of 984 (11.2\%) placebo recipients experienced $\geq 1$ treatment-emergent adverse event (TEAE), with no serious TEAEs reported in either group during this 48-h period (pooled ACHIEVE data; abstract) [23]. Within 30 days of any dose, $\geq 1$ TEAE was reported by $27.1 \%$ and $22.9 \%$ of ubrogepant and placebo recipients, with $9.4 \%$ and $8.9 \%$ of these TEAEs considered by the investigator to be treatment-related. Of the three serious TEAEs reported, none were considered treatmentrelated. Of the five cases of aspartate transaminase (AST)/ alanine transaminase (ALT) levels $\geq 3 \times$ the upper limit of normal (ULN) reported in the ubrogepant $50 \mathrm{mg}$ group, all were judged by an independent panel of liver experts blinded to treatment to be unlikely to be related to study treatment [23].

During the 48-h and 30-day postdose periods, there was no evidence of an increased risk of adverse events in ubrogepant recipients based on the presence or absence of cardiovascular (CV) risk factors at baseline in ACHIEVE I and II (pooled data), regardless of whether patients were categorized as having moderate to high, low or no $\mathrm{CV}$ risk (abstract) [24].

No safety concerns were identified during longer-term acute treatment of migraine attacks with ubrogepant $50 \mathrm{mg}$ or $100 \mathrm{mg}$, as required, in the 1-year extension study [25]. The safety population comprised 404, 409 and 417 patients in the ubrogepant $50 \mathrm{mg}$, ubrogepant $100 \mathrm{mg}$ and usual care groups, respectively; over the 1-year study period, 21,454 migraine attacks were treated with 31,968 doses of ubrogepant. TEAEs were reported by $66.3 \%$ and $72.6 \%$ of patients in the ubrogepant $50 \mathrm{mg}$ and $100 \mathrm{mg}$ groups during this period, with treatment-related adverse events reported by $10.4 \%$ and $10.5 \%$ of patients. One patient in the ubrogepant $50 \mathrm{mg}$ group experienced a serious adverse event (sinus tachycardia) that was considered to be treatment-related by the investigator; this patient had a history of supraventricular tachycardia with ablation. Of the 20 cases of AST/ ALT levels $\geq 3 \times$ ULN reported across treatment groups, two cases in the ubrogepant $50 \mathrm{mg}$ group were adjudicated by an independent panel of liver experts blinded to treatment to be possibly treatment-related and one case in the ubrogepant $100 \mathrm{mg}$ group was judged to be probably related (confounding factors were noted in this case). There were no cases of Hy's Law [25].

\subsection{Ongoing Clinical Trials}

There are currently no ongoing studies evaluating the efficacy of ubrogepant in the acute treatment of migraine. In September 2019, a randomized, open-label, phase I trial (NCT04179474) was initiated to evaluate the potential for a pharmacokinetic interaction and the safety and tolerability of ubrogepant when ubrogepant and erenumab or galcanezumab are coadministered (estimated completion date December 2019).

\section{Current Status}

On the 23 December 2019 [5], ubrogepant received its first global approval in the USA for the acute treatment of migraine ( \pm aura) in adults [6].

\section{Compliance with Ethical Standards}

Funding The preparation of this review was not supported by any external funding.

Conflict of interest During the peer review process the manufacturer of the agent under review was offered an opportunity to comment on the article. Changes resulting from any comments received were made by the authors on the basis of scientific completeness and accuracy. Lesley Scott is a salaried employee of Adis International Ltd/Springer Nature, is responsible for the article content and declares no relevant conflicts of interest. 
Open Access This article is distributed under the terms of the Creative Commons Attribution 4.0 International License (http://creativecommons.org/licenses/by/4.0/), which permits use, duplication, adaptation, distribution and reproduction in any medium or format, as long as you give appropriate credit original author(s) and the source, provide a link to the Creative Commons licence and indicate if changes were made.

\section{References}

1. Holland PR, Goadsby PJ. Targeted CGRP small molecule antagonists for acute migraine therapy. Neurotherapeutics. 2018;15(2):304-12.

2. Chan C, Goadsby PJ. Recent advances in pharmacotherapy for episodic migraine. CNS Drugs. 2019;33(11):1053-71.

3. Edvinsson L, Haanes KA, Warfvinge K, et al. CGRP as the target of new migraine therapies-successful translation from bench to clinic. Nat Rev Neurol. 2018;14(6):338-50.

4. Allergan, Merck. Allergan enters into licensing agreement with Merck to obtain exclusive worldwide rights to CGRP migraine development program [media release]. 7 Jul 2015.

5. US Food \& Drug Administration. FDA approves new treatment for adults with migraine [media release]. 23 Dec 2019. https:// www.fda.gov/news-events/press-announcements/fda-approvesnew-treatment-adults-migraine.

6. Allergan. UBRELVY (ubrogepant): US prescribing information. 2019. http://www.fda.gov. Accessed 13 Jan 2020.

7. Moore E, Burgey CS, Fraley M, et al. Characterization of ubrogepant: a potent and selective antagonist of the human calcitonin gene-related peptide receptor [abstract]. Headache. 2019;59(Suppl 1):89-90.

8. Edvinsson L, Rubio-Beltran E, Chan K, et al. Characterization of the effects of the calcitonin gene-related peptide (CGRP) receptor antagonists, atogepant and ubrogepant, on isolated human coronary, cerebral, and middle meningeal arteries [abstract no. OR13]. Headache. 2019;59(Suppl 1):13.

9. Jakate A, Boinpally R, Butler M, et al. Single therapeutic and supratherapeutic doses of ubrogepant do not affect cardiac repolarization in healthy adults: results from a randomized trial. Clin Pharmacol Ther. 2019. https://doi.org/10.1002/cpt.1696.

10. Li C, Palcza J, Xu J, et al. Absence of clinically significant drug interactions with coadministration of ubrogepant and an ethinyl estradiol/norgestimate oral contraceptive in healthy female subjects: a phase 1 pharmacokinetic analysis [abstract no. P100]. Headache. 2019;59(Suppl 1):91.

11. Jakate A, Boinpally R, Butler M, et al. Single therapeutic doses of ubrogepant are not associated with a clinically relevant drugdrug interaction when co-administered with acetaminophen or naproxen [abstract no. P96]. Headache. 2019;59(Suppl 1):87-8.
12. Jakate A, Boinpally R, Butler M, et al. Coadministration of single therapeutic oral doses of ubrogepant and sumatriptan produces no clinically relevant pharmacokinetic interactions [abstract no. P95]. Headache. 2019;59(Suppl 1):86-7.

13. Dodick DW, Lipton RB, Ailani J, et al. Ubrogepant for the treatment of migraine. N Engl J Med. 2019;381(23):2230-41.

14. Lipton RB, Dodick DW, Ailani J, et al. Effect of ubrogepant vs placebo on pain and the most bothersome associated symptom in the acute treatment of migraine: the ACHIEVE II randomized clinical trial. JAMA. 2019;322(19):1887-98.

15. Dodick DW, Goadsby PJ, Lakkis H, et al. Ubrogepant achieves onset of pain relief at 1 hour for the acute treatment of migraine [abstract no. P103]. Headache. 2019;59(Suppl 1):92-3.

16. Blumenfeld AM, Goadsby PJ, Dodick DW, et al. Ubrogepant is effective for the acute treatment of migraine in patients for whom triptans are ineffective [abstract no. IOR02]. Headache. 2019;59(Suppl 1):19.

17. Ailani J, Blumenfeld AM, Klein B, et al. An optional second dose of ubrogepant is effective in achieving 2-hour pain freedom in the acute treatment of migraine [abstract no. P112]. Headache. 2019;59(Suppl 1):98.

18. Voss T, Lipton RB, Dodick DW, et al. A phase IIb randomized, double-blind, placebo-controlled trial of ubrogepant for the acute treatment of migraine. Cephalalgia. 2016;36(9):887-98.

19. Li CC, Voss T, Kowalski K, et al. Making better dose decisions: using exposure-response modeling to integrate efficacy outcome of two phase $2 \mathrm{~b}$ clinical trials of ubrogepant for migraine treatment. Clin Transl Sci. 2019. https://doi.org/10.1111/cts.12730.

20. Lipton RB, Ailani J, Hutchinson S, et al. Efficacy is maintained with long-term intermittent use of ubrogepant for the acute treatment of migraine [abstract no. P135]. Headache. 2019;59(Suppl 1): 110 .

21. Ankrom W, Bondiskey P, Li CC, et al. Ubrogepant is not associated with clinically meaningful elevations of alanine aminotransferase in healthy adult males. Clin Transl Sci. 2020:1-11.

22. Goadsby PJ, Tepper SJ, Watkins PB, et al. Safety and tolerability of ubrogepant following intermittent, high-frequency dosing: randomized, placebo-controlled trial in healthy adults. Cephalalgia. 2019;39(14):1753-61.

23. Hutchinson S, Dodick DW, Treppendahl C, et al. Ubrogepant for the acute treatment of migraine: pooled safety and tolerability from ACHIEVE I and ACHIEVE II phase 3 studies [abstract no. P154]. Headache. 2019;59(Suppl 1):120.

24. Hutchinson S, Silberstein SD, Blumenfeld AM, et al. Safety of ubrogepant in participants with moderate to high cardiovascular risk [abstract no. P124]. Headache. 2019;59(Suppl 1):104-5.

25. Ailani J, Lipton RB, Hutchinson S, et al. Long-term safety evaluation of ubrogepant for the acute treatment of migraine; phase 3 , randomized, 52-week extension trial. Headache. 2020;60:141-52. 